\title{
Quantifying the light parton transport properties with jet and hadron $R_{A A}$
}

\author{
Tianyu Dai, ${ }^{a, *}$ Steffen A. Bass, ${ }^{a}$ Jean-François Paquet $^{a}$ and Derek Teaney ${ }^{b}$ \\ ${ }^{a}$ Department of Physics, Duke University, \\ Physics Blvd. Science Dr., Durham NC 27707, United States \\ ${ }^{b}$ Department of Physics and Astronomy, Stony Brook University \\ Stony Brook, New York 11794, United States \\ E-mail: tianyu.dai@duke.edu, bass@duke.edu, jeanfrancois.paquet@duke.edu, \\ derek.teaney@stonybrook.edu
}

Interactions between hard partons and the quark-gluon plasma range from frequent soft interactions to rare hard interactions. While it is reasonable that hard interactions can be described perturbatively, soft interactions likely have significant non-perturbative effects. The cumulative effect of the soft interactions can be encoded into parton transport coefficients, which can be constrained from heavy ion measurements. In this work, we perform a simplified proof of principle calculations showing that the magnitude of the drag and diffusion of light partons can be constrained from the hadronic and jet $R_{A A}$, assuming a known temperature dependence of the soft transport coefficients. We show how this study can be implemented naturally in a parton energy loss model that factorizes systematically soft and hard partonic interactions. We use this proof of principle calculation to understand how reducing uncertainties on $R_{A A}$ can improve constraints on the parton's transport coefficients. We also observe the complementarity of jet and hadronic observables to constrain the light parton transport coefficients.

Hard Probes 2020

Online

1-6 June 2020

\footnotetext{
${ }^{*}$ Speaker
} 


\section{Introduction}

The QCD plasma produced in heavy ion collisions is strongly coupled, and its internal dynamics is non-perturbative. Nonetheless the interactions of energetic parton with this plasma may still be treated perturbative, given a sufficiently large momentum transfer between the parton and the plasma. For interactions with smaller momentum transfer — "soft interactions" - non-perturbative effects are likely significant. A parton energy loss model which systematically factorizes hard and soft interactions was introduced in Refs. [1-4]. Soft interactions are described stochastically with lightparton drag and the diffusion coefficients. These "soft" transport coefficients can be parametrized and constrained from measurements, making it possible to quantify non-perturbative effects.

Before using this approach with experimental measurements, we perform a proof of principle calculation, or "closure test": we evaluate a set of high- $p_{T}$ observables using our model, and study the constraining power of these observables within this controlled environment. Using this approach, we can quantify systematically the extent to which the transport coefficients of light partons can be constrained from the jet and hadronic $R_{A A}$.

\section{Hard-soft factorized model of parton energy loss}

The parton energy loss model used in this work is the hard-soft factorized model introduced in Refs. [1-4]. This hard-soft factorized model has its root in the weakly-coupled effective kinetic approach [5], in which quarks and the gluons can be described by Boltzmann transport equations as quasi-particles with effective masses and cross-sections.

Leading order realizations based on the weakly-coupled effective kinetic approach (e.g. MARTINI [6]) divide the interactions as (i) $2 \leftrightarrow 2$ elastic interactions, and (ii) effective $1 \leftrightarrow 2$ inelastic interactions. In the hard-soft factorized model, the elastic and inelastic interactions are further divided as hard and soft parton-plasma interactions. The large number of the soft elastic and inelastic interactions are described stochastically as a diffusion process with parton transport coefficients. Rare hard interactions are handled with perturbative collision rates. The diffusion process does not rely on the quasi-particle assumption, and thus can be extended to the phenomenological largecoupling regime. The simpler form of the soft transport coefficients, which can effectively depend only on temperature $T$, is also well suited for data-driven constraints.

We numerically implement this hard-soft factorized model in the JETSCAPE framework [7]. In the numerical implementation, we set the hard-soft cutoffs $\mu_{\tilde{q}_{\perp}}$ and $\mu_{\omega}$ on the $\tilde{q}_{\perp}$ and $\omega$ to divide the phase space of the interactions into the hard interactions and soft interactions, where $\tilde{q}_{\perp} \equiv \sqrt{q^{2}-\omega^{2}}, q$ is the momentum exchange, and $\omega$ is the energy exchange [3]. In this way, the $2 \leftrightarrow 2$ elastic interactions and $1 \leftrightarrow 2$ inelastic interactions are reformulated as hard interactions treated using collision rates and a diffusion process realized as a Langevin model.

In the small coupling regime, the soft drag and diffusion coefficients in the diffusion process can be calculated analytically using perturbation theory:

$$
\begin{gathered}
\hat{q}_{L, \text { soft }}^{2 \leftrightarrow 2, \text { analytical }}=\frac{g^{2} C_{R} T M_{\infty}^{2}}{4 \pi} \ln \left[1+\left(\frac{\mu_{\tilde{q}_{\perp}}}{M_{\infty}}\right)^{2}\right], \quad \hat{q}_{\text {soft }}^{2 \leftrightarrow, \text { analytical }}=\frac{g^{2} C_{R} T m_{D}^{2}}{4 \pi} \ln \left[1+\left(\frac{\mu_{\tilde{q}_{\perp}}}{m_{D}}\right)^{2}\right] \\
\hat{q}_{L, \text { soft }}^{1 \leftrightarrow 2 \text {,analytical }}=\frac{(2-\ln 2) g^{4} C_{R} C_{A} T^{2} \mu_{\omega}}{4 \pi^{3}},
\end{gathered}
$$


where $C_{R}$ is the Casimir factor, $m_{D} \equiv g T \sqrt{N_{c} / 3+N_{f} / 6}$ is the leading order Debye mass, $M_{\infty} \equiv$ $\sqrt{m_{D}^{2} / 2}$ is the gluon asymptotic thermal mass, $N_{c}$ is the number of the quark color, $N_{f}$ is the number of the quark flavor.

These transport coefficients encode the effect of soft interactions only. Thus these expressions likely do not hold at large coupling and will need to be constrained from experimental data. Here we have assumed that the momentum transfer of these soft interactions are always much smaller than the energetic parton's momentum, hence the absence of momentum dependence in Eqs. 1-2. This is different from definitions of $\hat{q}$ which include the effect of all interactions and thus have a momentum dependence.

\section{Proof of principle calculation: closure test in Bayesian inference}

The proof of principle calculation is performed as follows. We fix the hard-soft cutoff to $\mu_{\omega}=\mu_{\tilde{q}_{\perp}}=2 T$ and keep it fixed for the current analysis. All interactions above this cutoff (the hard interactions) are given by the usual (unscreened) $2 \leftrightarrow 2$ matrix elements, and $1 \leftrightarrow 2$ rates. We assume that the temperature dependence of the soft transport coefficients is the same as the perturbative one (Equation 1-2) and we vary only their normalization:

$$
\hat{q}_{\text {soft }}^{2 \leftrightarrow 2}=\kappa \hat{q}_{\text {soft }}^{2 \leftrightarrow 2, \text { analytical }} ; \hat{q}_{L, \text { soft }}^{2 \leftrightarrow 2}=\kappa \hat{q}_{L, \text { soft }}^{2 \leftrightarrow 2, \text { analytical }} ; \hat{q}_{L, \text { soft }}^{1 \leftrightarrow 2}=\kappa \hat{q}_{L, \text { soft }}^{1 \leftrightarrow 2, \text { analytical }}
$$

The goal here is thus to see how well the data can constrain the normalization $\kappa$, which reflects the most strongly coupled part of the parton energy loss calculation arising from soft interactions.

We set $\kappa=\tilde{\kappa}$ in Eq. 3 (typically we take $\tilde{\kappa}=1$ ), and then calculate the jet and hadron $R_{A A}$. These calculations are treated as if they were measurements; we refer to them as "constructed data" and denote them as $\tilde{R}_{A A}^{j e t / h}$. We separately calculate the jet and hadron $R_{A A}$ for a range of possible values of $\kappa$ between 0.2 and 2.0. This defines our "parameter space": the range of parameters (or, equivalently, of transport coefficients) that we want to explore in this work. We then use an emulator/interpolator to estimate the values of the jet and hadron $R_{A A}$ observables for any values of $\kappa$ between 0.2 and 2.0. Finally, we use this emulator $R_{A A}^{j e t / h}(\kappa)$ to perform a Bayesian parameter estimation on the "constructed data" $\tilde{R}_{A A}^{j e t / h}$, and verify if we can recover $\tilde{\kappa}$.

The results of the Bayesian parameter estimation depends on the uncertainties assumed in the analysis. We assume that both experimental and theoretical uncertainties are normally distributed. We also assume that the observables are not correlated. In this case, the posterior takes the form

$$
P\left(\tilde{R}_{A A}^{j e t / h}, \kappa\right) \propto \exp \left(-\chi^{2} / 2\right) ; \quad \chi^{2}=\sum_{s=j e t, h} \frac{\left(\tilde{R}_{A A}^{s}-R_{A A}^{s}(\kappa)\right)^{2}}{\left(\left(\delta \tilde{R}_{A A}^{s}\right)^{2}+\left(\delta R_{A A}^{s}(\kappa)\right)^{2}\right)}
$$

where $\delta \tilde{R}_{A A}^{s}$ are the uncertainties on the "constructed data", and $\delta R_{A A}^{s}(\kappa)$ are the uncertainties of the calculations.

\section{Results}

We use $\kappa=\tilde{\kappa}=1$ to evaluate the "constructed " $\tilde{R}_{A A}^{j e t / h}$ for both parton jets and charged hadrons using a single hydrodynamic event. The temperature depence of $\hat{q}_{\text {soft }}^{2 \leftrightarrow 2}$ is shown by the 

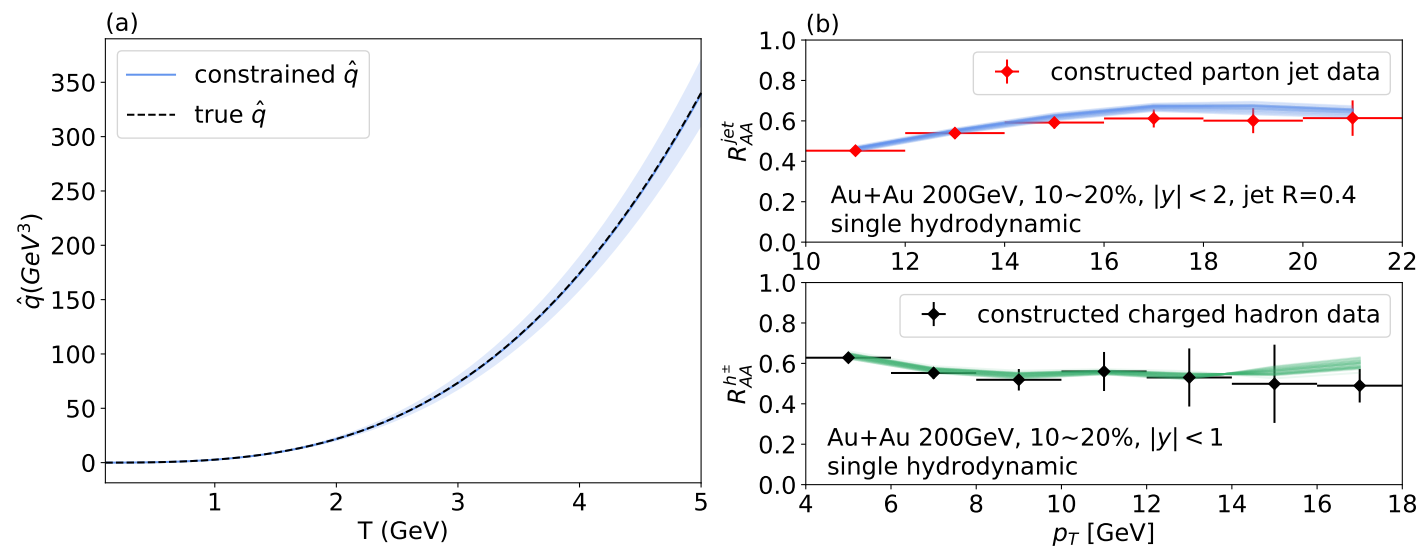

Figure 1: (a) The temperature dependence of $\hat{q}_{\text {soft }}^{2 \leftrightarrow 2}$ and the $95 \%$ confidence interval of $\hat{q}_{\text {soft }}^{2 \leftrightarrow 2}$ after Bayesian analysis; the $T^{3}$ dependence is fixed, and thus the figure indicates the quality of the constrained normalization. (b) The "constructed data" and the sampled data using the constrained parameter.
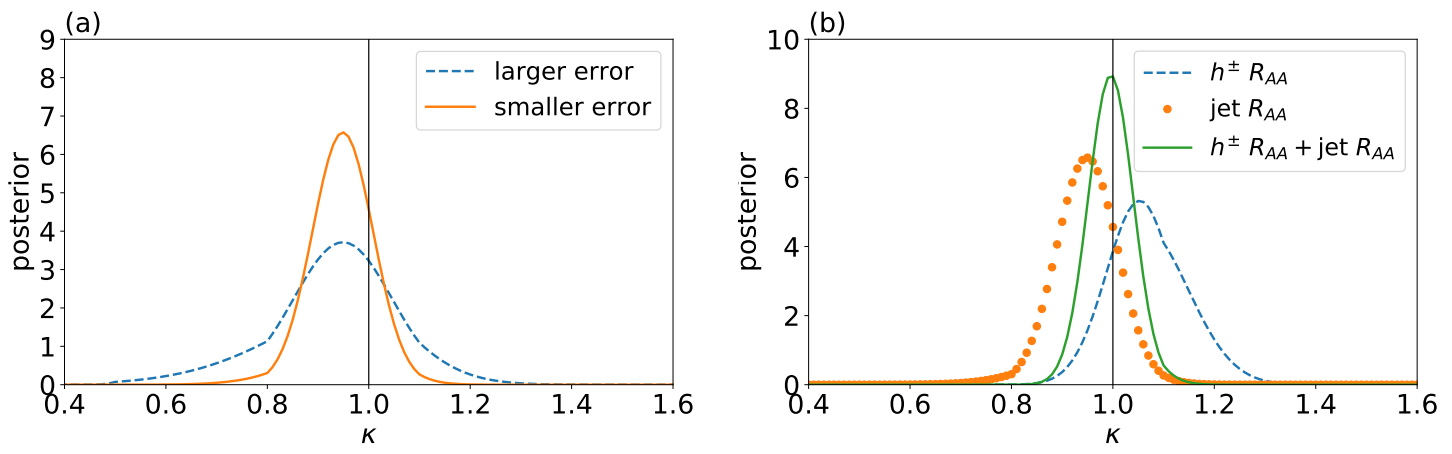

Figure 2: (a) the posterior distribution of $\kappa$ using different data uncertainties; (b) the posterior distribution of $\kappa$ using different observables.

dashed line in Figure 1(a), while resulting jet and hadron $R_{A A}$ are shown by the points in Figure 1(b). The uncertainties used in the analysis $-\delta \tilde{R}_{A A}^{s}$ and $\delta R_{A A}^{s}(\kappa)$ in Eq. $4-$ are proportional to the statistical uncertainties from the calculations of the jet and hadron $R_{A A}$ : for $\delta R_{A A}^{s}(\kappa)$, we used the statistical uncertainties, while for $\delta \tilde{R}_{A A}^{s}$, we multiplied these statistical uncertainties by a factor of three such that the uncertainties on the "constructed data" are closer to those encountered in actual measurements.

With the above values for (i) the model predictions; (ii) the "constructed data" $\tilde{R}_{A A}^{s}$; and (iii) the uncertainties, we evaluate the posterior distribution for the normalization $\kappa$ of the $\hat{q}$. We first only use the jet $R_{A A}$ as the "constructed data". The coefficient $\kappa$ is constrained around $\tilde{\kappa}=1$ as shown in the narrower curve in Figure 2(a). To explore the effect of uncertainties on the Bayesian inference, we increase the uncertainty on the "constructed data" by $66 \%$ and recalculate the posterior of $\kappa$. We plot the posterior distribution of $\kappa$ generated with the larger data uncertainty as the wider curve in Figure 2(a). The width of the posterior increases by approximately the same factor as the change in the uncertainty of $R_{A A}$.

We also quantify the contribution of each observable to constrain the normalization of soft transport coefficients $\kappa$. We plot the posterior distribution of $\kappa$ for three different scenarios: (i) 
using only the hadronic $R_{A A}$; (ii) using only the jet $R_{A A}$; or (iii) using both the hadronic and jet $R_{A A}$. The posterior is plotted in Figure 2(b). With only the hadronic $R_{A A}$ or only the jet $R_{A A}$, the full width at the half maximum of the posterior distribution is 0.17 and 0.14 . With both jet $R_{A A}$ and hadronic $R_{A A}$, the width of the posterior is decreased to 0.11 , and the peak is closer to $\tilde{\kappa}=1$. Given the posterior distribution of $\kappa$, we show the $95 \%$ confidence interval of $\hat{q}_{\text {soft }}^{2 \leftrightarrow 2}$ as a band in Figure $1(\mathrm{a})$; it is fully consistent with the true value of the soft transport coefficients used to evaluate the reference $\tilde{R}_{A A}^{s}$.

As a final verification, we sample $\kappa$ using the combined posterior distribution in Figure 2(b) and evaluate both the hadronic and jet $R_{A A}$ at these samples. The result is shown as a band in Figure 1(b): it is found to be consistent with the input "constructed" data, as expected.

\section{Conclusion \& outlook}

We performed a proof of principle calculation to constrain the magnitude of the soft transport coefficients of hard partons, assuming that they have a simple and known temperature dependence. Parton jet $R_{A A}$ and charged hadron $R_{A A}$ were found to provide complementary information that allows for better constraints on the soft transport coefficients. In the bigger picture, our result suggests that constraining the normalization of the soft parton coefficient is straightforward even with limited measurements, assuming a strong knowledge of the temperature dependence of the soft transport coefficients. We intend to confirm these results by repeating the analysis with (i) a more flexible temperature dependence of soft transport coefficients; (ii) additional jet observables; and (iii) allowing the hard-soft cutoffs to vary in a range of reasonable values.

Acknowledgments This work was supported by the U.S. Department of Energy Grant no. DEFG02-05ER41367 (SAB, JFP and TD) and DE-FG-02-08ER41450 (DT). TD is also supported by NSF grant OAC-1550225.

\section{References}

[1] J. Ghiglieri, G. D. Moore and D. Teaney, Jet-medium interactions at NLO in a weakly-coupled quark-gluon plasma, Journal of High Energy Physics 2016 (2016) 95.

[2] T. Dai, S. A. Bass, J.-F. Paquet and D. Teaney, Parton energy loss in the reformulated weakly-coupled kinetic approach, Proceedings of Science - HardProbes2018 (2018) 63.

[3] T. Dai, S. A. Bass, J.-F. Paquet and D. Teaney, Hard scattering and stochastic reformulation of parton energy loss, Proceedings of Science - High-pT2019 (2019) 31.

[4] J. Ghiglieri and D. Teaney, Parton energy loss and momentum broadening at NLO in high temperature QCD plasmas, International Journal of Modern Physics E 24 (2015) 1530013.

[5] P. B. Arnold, G. D. Moore and L. G. Yaffe, Effective kinetic theory for high temperature gauge theories, Journal of High Energy Physics 2003 (2003) 030.

[6] B. Schenke, C. Gale and S. Jeon, MARTINI: an event generator for relativistic heavy-ion collisions, Phys. Rev. C80 (2009) 054913 [0909. 2037].

[7] J. Putschke, K. Kauder, E. Khalaj, A. Angerami, S. Bass, S. Cao et al., The JETSCAPE framework, arXiv preprint arXiv:1903.07706 (2019) . 